\title{
Lupus Nephritis and Chronic Kidney Disease
}

Renal involvement in systemic lupus erythematosus (SLE) occurs in $40-50 \%$ of adult patients, is associated with increased morbidity and mortality, and results in endstage renal disease (ESRD) about $10 \%$ of patients - rates that have changed little over the last 2 decades despite modifications in therapeutic strategies ${ }^{1}$.

In all of us there is a gradual decline in kidney function that occurs with age, with estimates of losses of 0.4-1 $\mathrm{ml} / \mathrm{min} /$ year in those over 30 years of age being reported ${ }^{2,3}$. Assuming a normal glomerular filtration rate (GFR) of 100 $\mathrm{ml} / \mathrm{min}$ in early adulthood and assuming no changes in this rate due to intercurrent illnesses, our kidney function is sufficient to last a lifetime. However, in the presence of renal disease, for example, diabetic nephropathy or polycystic kidney disease, rates of loss of kidney function can be 10 -fold greater or more, in turn meaning there is a significant chance of needing renal replacement therapy in the form of dialysis or transplantation during a patient's lifetime. In addition, modest reductions in GFR are associated with significant increases in overall and specific cardiovascular mortality. We know that certain interventions can slow down this rate, blood pressure control being the main factor, and specific interventions such as good glycemic control and novel sodium-glucose linked transporter (SGLT) 2 inhibitors for diabetes, or vasopressin receptor antagonists for polycystic kidney disease, respectively. What about other conditions where kidney decline is not a continuous process but has a more episodic character, such as occurs in relapsing lupus nephritis ( $\mathrm{LN})$ ? The aim of treatment in $\mathrm{LN}$ is to halt and reverse the current renal inflammation to reverse acute kidney dysfunction but also prevent scarring (an irreversible loss of nephrons, the functional unit of the kidney) and development of chronic kidney disease (CKD). This is important, because evidence suggests that the compensatory response to a reduction in functional nephron mass, whatever the primary cause, can in itself be damaging and lead to further scarring, and instigate a viscous cycle of renal function decline.

CKD has been defined by different stages of severity from 1 to 5, based on the estimated GFR (eGFR) calculated using serum creatinine (or cystatin $\mathrm{C}$ ) and equations
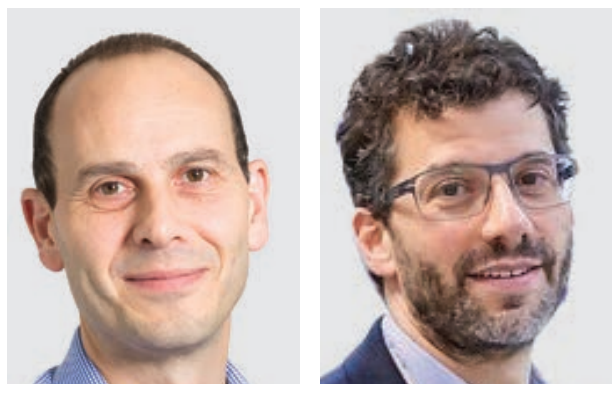

that have been validated for certain populations. There are many factors that contribute to progressive CKD and can be broadly grouped under different pathogenic mechanisms (Table 1), including the starting nephron endowment, the renal insult, and the resulting hemodynamic or inflammatory environment. In addition, there are clear genetic factors that influence rates of CKD progression, as demonstrated by the disparities between prevalence of CKD and incidence of ESRD in different ethnic groups ${ }^{4}$.

Data from the Chronic Renal Insufficiency Cohort (CRIC) study suggest that, alongside well-known hemodynamic risk factors such as hypertension, patients who progress the most rapidly have greater levels of inflammation, as demonstrated by raised levels of tumor necrosis factor (TNF)- $\alpha$ or interleukin (IL) $6^{5}$. This implies that ongoing inflammation may not be adequately recognized, leading to subclinical renal injury that may in turn be a significant contributor to CKD progression. We know that our biomarkers of disease activity, especially in diseases such as LN, are poor and this has resulted in a need for repeated biopsies in many patients, where urinary or serological markers do not inform sufficiently. Novel biomarkers are coming into clinical practice and their utility in real-world settings will need to be seen ${ }^{6}$.

Table 1. Pathogenic mechanisms that contribute to progressive CKD.

\begin{tabular}{ll}
\hline $\begin{array}{l}\text { Mechanism Underlying } \\
\text { CKD Progression }\end{array}$ & Variables \\
\hline $\begin{array}{l}\text { Nephron loss (acquired or } \\
\text { hereditary) }\end{array}$ & $\begin{array}{l}\text { Birth prematurity, genetic factors, } \\
\text { primary renal disease, age, episodes } \\
\text { of AKI, nephrotoxins } \\
\text { Glomerular hypertension, systemic } \\
\text { blood pressure, glomerular } \\
\text { hemodynamic responses to }\end{array}$ \\
hyflammation & $\begin{array}{l}\text { Circulating or renal } \\
\text { proinflammatory cytokines } \\
\text { (e.g., TNF- } \alpha, \text { IL-6) } \\
\text { Profibrotic cytokines (e.g., TGF- } \alpha, \\
\text { AGE, angiotensin II) }\end{array}$ \\
\hline
\end{tabular}

CKD: chronic kidney disease; AKI: acute kidney injury; TNF: tumor necrosis factor; IL: interleukin; TGF: transforming growth factor; AGE: advanced glycation end products.

See Advanced CKD in lupus nephritis, page 1366 
In this issue of The Journal, Tselios, et al report on a retrospective analysis on the progression of CKD in a cohort of 118 Canadian patients with LN and CKD (stages 3b-4), followed for 10 years 7 . The authors ask whether dialysis (ESRD) is inevitable. The cohort consisted of 118 patients out of a total of 700 with LN enrolled in their registry from 1970 onward. They show that only 38\% of patients progressed from stages $3 \mathrm{~b}$ to 4 or 5 , or from 4 to 5 . This is consistent with a wide range of studies that report CKD progression in non-LN populations can be slowed using a variety of measures, and indeed in older patients CKD decline may flatten for a number of years.

So what does this paper tell us? Clearly, treating patients with LN and CKD is still of value because only $38 \%$ of their patients progressed, and markers of disease activity, albeit imperfect ones, were associated with CKD progression, reinforcing the idea that active inflammation in the kidney will result in further irreversible damage.

It is important to note that complement levels and dsDNA antibody titers were abnormal in over a third of the non-progressors, highlighting the inadequacy of our current biomarkers. In addition, treatment of CKD with renin-angiotensin-aldosterone system (RAAS) blockers was associated with less progression, already well established in various proteinuric kidney diseases, although interestingly, the level of proteinuria (a predictor of CKD progression in almost every existing study to have examined this) was not associated with outcome. Importantly for an $\mathrm{LN}$ population discussing progression of CKD, two-thirds of the cohort were white, less than $20 \%$ were black, and ethnicity was also not associated with different outcomes.

However, this study also highlights some of the challenges of conducting studies of renal decline ${ }^{7}$. Inclusion criteria required 2 consecutive low eGFR measurements, but those with more aggressive disease are likely to be seen more regularly and may meet this criterion even if they go on to recover renal function. Further, the definition of progression by change in CKD stage is in itself unlikely to be important to patients and will provide less insight than examining eGFR trajectories over time directly.

What does the paper not tell us? There is no consideration of non-compliance, which is known to be an important factor in patients with SLE and is a predictor of poor outcomes. There is a paucity of information regarding the induction therapy (missing in over half the patients), which is also important in defining response and hence progression of $\mathrm{LN}$. There is no description of intervening episodes of acute kidney injury, a key risk factor for CKD progression, and a consequence not only of disease relapse but also of infection, which is in turn more common in those receiving augmented immunosuppression. In addition, patients were treated over 4 decades, during which various changes to management of LN and CKD will have been instituted (improved use of erythropoietin, moving away from calcium-based phosphate binders that contribute to vascular calcification, as well as specific changes in LN management).
The authors believe that highlighting these data should encourage LN patients with CKD to be entered into clinical trials, from which they have been historically excluded ${ }^{7}$. This would be a valuable addition to the therapeutic outcome data from trials; however, the clinical endpoint would have to be reconsidered, because many that are currently used would not be suitable. Proteinuria and hematuria may represent scarred glomeruli or actively inflamed glomeruli, and recovery of creatinine levels or eGFR will not be the same in patients with preexisting CKD and those with previously normal kidney function. So careful consideration of trial endpoints will be needed if such patients are included, and at the moment there is a paucity of good markers to confirm silencing of renal inflammation in this group of patients.

That CKD progression is not inevitable and that patients with $\mathrm{LN}$ who have developed CKD do not always progress to ESRD is reassuring. It means we must continue to aggressively treat LN so as to modify some of the critical factors leading to renal damage, which include not only the immunosuppression we use, but other strategies: strict blood pressure control (ideally using RAAS blockade), treatment of concurrent diabetes (and potentially glycosuric agents, such as SGLT2 inhbitors in the non-diabetic population), cessation of smoking, and consideration of other cardiovascular risk factors.

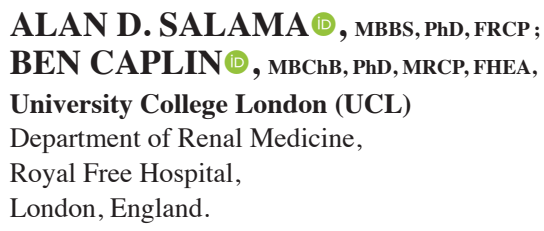

Address correspondence to A.D. Salama, Royal Free Hospital, UCL Centre for Nephrology, Rowland Hill Street, London NW3 2PF, UK. E-mail: a.salama@ucl.ac.uk, alan.salama@nhs

\section{REFERENCES}

1. Almaani S, Meara A, Rovin BH. Update on lupus nephritis. Clin J Am Soc Nephrol 2017;12:825-35.

2. KDIGO. Clinical practice guideline for the evaluation and management of chronic kidney disease. Kidney Int Suppl 2013;3:63-72.

3. Fenton A, Montgomery E, Nightingale P, Peters AM, Sheerin N, Wroe AC, et al. Glomerular filtration rate: new age- and gender- specific reference ranges and thresholds for living kidney donation. BMC Nephrol 2018;19:336.

4. Hsu CY, Lin F, Vittinghoff E, Shlipak MG. Racial differences in the progression from chronic renal insufficiency to end-stage renal disease in the United States. J Am Soc Nephrol 2003;14:2902-7.

5. Amdur RL, Feldman HI, Gupta J, Yang W, Kanetsky P, Shlipak M, et al. Inflammation and progression of CKD: the CRIC study. Clin J Am Soc Nephrol 2016;11:1546-56.

6. Mejia-Vilet JM, Zhang XL, Cruz C, Cano-Verduzco ML, Shapiro JP, Nagaraja HN, et al. Urinary soluble CD163: a novel noninvasive biomarker of activity for lupus nephritis. J Am Soc Nephrol 2020 Apr 16 (E-pub ahead of print).

7. Tselios K, Gladman DD, Jiandong S, Urowitz MB. Advanced chronic kidney disease in lupus nephritis: is dialysis inevitable? J Rheumatol 2020;47:1366-73.

J Rheumatol 2020;47:1303-4; doi:10.3899/jrheum.200566

Personal non-commercial use only. The Journal of Rheumatology Copyright $(\subset) 2020$. All rights reserved. 